\title{
Impacto de la Enseñanza de la Geometría Descriptiva usando Archivos 3D-PDF como Entrenamiento de la Habilidad Espacial de Estudiantes de Ingeniería Civil en el Perú
}

\author{
Hugo C. Gómez-Tone ${ }^{(1,2)}$ \\ (1) Facultad de Arquitectura y Urbanismo, Universidad Nacional de San Agustín de Arequipa, Perú. \\ (e-mail: hgomezt@unsa.edu.pe) \\ (2) Facultad de Ingenierías, Universidad Católica San Pablo, Urb. Campiña Paisajística, Arequipa, Perú.
}

Recibido May. 15, 2018; Aceptado Jul. 23, 2018; Versión final Ago. 17, 2018, Publicado Feb. 2019

\begin{abstract}
Resumen
El objetivo de esta investigación es determinar la mejora de la capacidad espacial de los estudiantes de ingeniería a través de la enseñanza del curso denominado Geometría Descriptiva apoyado con modelos 3DPDF. Esta capacidad es trascendente para los que pretenden estudiar ingeniería, pero sin consenso en cuanto al mejor tipo de entrenamiento. En este estudio se midió la capacidad espacial de 216 estudiantes (muestra no probabilística intencional) de la carrera de Ingeniería Civil de la Universidad Católica de Santa María y de la Universidad Católica San Pablo de Arequipa. Se usó el Test de Visualización Espacial Purdue: rotaciones, antes y después del curso y se aplicó las pruebas estadísticas t de Student y U de Mann-Whitney. Se encontró una mejora moderada del orden del $22 \%$. Estos resultados sugieren que el entrenamiento indirecto de la capacidad espacial, a través de los cursos de graficación mejorados con tecnologías digitales, pueden convertirse en herramientas de enseñanza eficientes.
\end{abstract}

\section{Impact of Descriptive Geometry's Teaching using 3D-PDF Files as Spatial Skill Training in Civil Engineering Students in Peru}

\begin{abstract}
The main purpose of this research is to determine de Spatial Skill enhance in engineering students through the teaching of Descriptive Geometry subject supported by 3D-PDF models. This skill is of paramount importance for all people who pretend to study engineering. However, there is not agreement about the best training. In this research, it was assessed the Spatial Skill of 216 students (non-probabilistic sample) from Civil Engineering at the Saint Mary Catholic University and Saint Pablo Catholic University from Arequipa using the Purdue Spatial Visualization Tests: rotations, before and after the training, then the t-Student test and the $U$ test of Mann-Whitney were applied. It was found a moderate improvement of the order of $22 \%$. These results suggest that the indirect training of the Spatial Skill through engineering graphics subjects, enhanced and supported with digital technologies, can become efficient teaching tools.
\end{abstract}

Keywords: spatial skill; engineering; training; descriptive geometry 


\section{INTRODUCCIÓN}

Uno de los cursos que más les cuesta superar a algunos estudiantes de las carreras de ingenierías en su primer año de estudios es la Geometría Descriptiva porque esta materia desarrolla y evalúa de manera indirecta la inteligencia espacial. En el Perú no se conoce la trascendencia de evaluar y desarrollar esta inteligencia en los futuros ingenieros. El propósito de esta investigación es identificar y comparar el nivel inicial de capacidad espacial de los alumnos que comienzan sus estudios universitarios de ingeniería civil y el nivel final al término del primer curso de Geometría Descriptiva, apoyado con una herramienta de visualización de modelos tridimensionales en tiempo real (archivos 3D-PDF).

La capacidad espacial es un componente de la inteligencia humana que nos permite formar, reconocer y manipular imágenes, figuras y objetos mentalmente (Arrieta, 2006). Esta capacidad integra tres aspectos importantes: primero está la aptitud, es decir, el talento innato recibido de manera hereditaria, luego la habilidad, que es la facilidad y posibilidad de aprenderse a través del entrenamiento (aspecto fundamental para este estudio) y finalmente la destreza que permite ejecutar las anteriores (Saorín-Pérez et al., 2009). De otro lado, la estructura de esta capacidad espacial, en función del proceso mental realizado al momento de solucionar una tarea de visualización, considera dos componentes (Tartre, 1990): La orientación espacial que consiste en mover nuestro punto de visión mentalmente en torno a un objeto que permanece fijo en el espacio, y la visualización espacial que consiste en mover mentalmente un objeto en el espacio sin implicar algún movimiento del observador. Este último es el componente más estudiado y tiene a su vez dos subcomponentes, la transformación mental en la que la percepción del objeto cambia debido a un cambio de forma del objeto por acciones de doblado, corte u otros y la rotación mental en la que la percepción del objeto cambia debido a giros del objeto en el espacio. La rotación mental es el subcomponente "vital" (Kösa y Karakuş, 2018) en la visualización espacial y el que tiende a mostrar las más grandes diferencias individuales y de género (Maeda y Yoon, 2013) además de ser el más relacionado con las investigaciones conducentes a su relación con las ingenierías (Sorby, 1999), por lo tanto, es esta investigación se utilizan pruebas que miden la rotación espacial para efectos de conocer la capacidad espacial.

Desde hace un siglo, se ha sostenido de manera continua y longitudinal que la capacidad espacial es un atributo y una característica muy importante que debe tener toda persona interesada en incurrir con éxito en el campo de las ingenierías, matemática, ciencias y tecnologías (Wai et al., 2009). En el caso específico de las ingenierías, se ha demostrado que la capacidad espacial tiene una relación directa con las tasas de retención de los ingresantes universitarios en dichas carreras y con el mejor rendimiento en los cursos de naturaleza gráfica (Sorby y Baartmans, 2000), pero además también con el éxito en otros cursos del primer año de estudios en las carreras de ingeniería (Sorby et al., 2014). Sin embargo su importancia va más allá del impacto de esta capacidad en el éxito de los futuros ingenieros sino fundamentalmente en el desarrollo tecnológico de un país, lo que ha generado por ejemplo, que los Estados Unidos de Norteamérica impulse de manera muy intensa la evaluación y desarrollo de esta capacidad desde hace décadas y con mayor énfasis en este milenio; como ejemplo se tiene el documento denominado "Preparando la Próxima Generación STEM de Innovadores: Identificando y Desarrollando el Capital Humano de Nuestra Nación" (CEH, 2010) el que recomendó continuar y ampliar la evaluación de las tres principales capacidades necesarias para los talentos en dichas carreras como son la capacidad matemática, la verbal y con especial atención la capacidad espacial antes muy descuidada. También, un año antes se lanzó la "ENGAGE Engineering" una Fundación Nacional para la Ciencia cuya finalidad es retener a estudiantes de pregrado de ingenierías al implementar tres estrategias dentro de las cuales una es el entrenamiento de las habilidades para la visualización espacial, dicha fundación tiene afiliadas actualmente 72 escuelas de ingeniería de ese país (Metz, 2015).

En nuestro país, no se ha dado la importancia necesaria a la evaluación y desarrollo de la capacidad espacial ni en la educación básica ni en la superior. De esta forma, las universidades están recibiendo estudiantes de ingeniería con muy variadas capacidades espaciales debido a la falta de evaluación de estas en los procesos de admisión (Segil et al., 2017). Ocurre entonces, que en el primer año los estudiantes de ingeniería con mayor capacidad espacial obtienen mayor nota en los cursos de graficación (Arrieta y Medrano, 2015) y, en general, en los demás cursos "clave" como el cálculo, física o química, mientras que los estudiantes con un pobre desarrollo de dichas capacidades se encuentran en gran desventaja al afrontar estos cursos (Acevedo et al., 2015) y los de graficación (Sorby, 2007).

La capacidad espacial puede desarrollarse con entrenamiento, el cual según Baenninger y Newcombe (1989), resulta ser más efectivo cuando es específico, es decir, corto (dos o tres semanas) y orientado a un test específico de visualización espacial, como también ha sido demostrado en la práctica a través de innumerables investigaciones, destacando las realizadas en la Universidad Tecnológica de Míchigan desde el año 1993 (Sorby, 2007). Sin embargo los entrenamientos generales o indirectos, que coinciden en la mayoría de casos con la instrucción de cursos obligatorios de graficación en ingenierías, también han mostrado efectividad en el desarrollo de esta capacidad, pero en menor grado a pesar de su más larga 
duración (Baenninger y Newcombe, 1989). Dada esta diferencia y la incursión de las tecnologías de información y comunicación en la educación, es que en las últimas dos décadas se han hecho muchas investigaciones buscando hacer más efectivo el entrenamiento indirecto apoyando la enseñanza de los cursos de Dibujo Técnico y la Geometría Descriptiva con entornos 3D multimedia y web, softwares especializados, videojuegos, realidad virtual, realidad aumentada, prototipado rápido, etc. Dentro de las muchas investigaciones al respecto, se ha encontrado al menos tres tendencias: en la primera están los que consideran que la inclusión de las tecnologías informáticas como el computador, entornos virtuales y otros permite un aprendizaje mayor de la habilidad espacial (Contreras et al., 2013) que los métodos tradicionales considerados insuficientes (Alqahtani et al., 2017); en la segunda están los que sostienen que las técnicas tradicionales como el dibujo a mano (Pieterse y Nel, 2013) o la manipulación física de objetos (Tristancho et al., 2014) mejoran las capacidades espaciales y que solo luego ayudan a complementarlas los programas de diseño y dibujo asistido por computadora (Pieterse y Nel, 2013) los cuales no son considerados útiles en el entrenamiento inicial (Sorby, 2009) y en la última tendencia están los que afirman que la combinación de la ingeniería gráfica tradicional con las tecnologías informáticas (el modelado y visualización tridimensional) favorecen en mayor medida el desarrollo de la capacidad espacial (Leopold et al., 2001; Marunić y Glažar, 2014).

Dadas estas tendencias, el presente estudio pretende encontrar más evidencias que fortalezcan esta última, sin embargo el aporte significativo recae en el diseño de modelos 3D-PDF para cada tema propio de la Geometría Descriptiva, comenzado por los diferentes sistemas de representación de objetos, pero fundamentalmente para los temas más abstractos de esta asignatura como es el estudio del punto, la recta, el plano y sus diferentes relaciones métricas (véase figuras 2, 3 y 4), aspectos que no se han encontrado en otros estudios, concentrándose estos en la visualización de piezas volumétricas y sus sistemas de representación.

\section{MÉTODOLOGÍA}

En este estudio, los métodos son abordados caracterizando a los participantes (sujetos de estudio para esta investigación), los instrumentos (test aplicado), el procedimiento (como se efectuó el estudio) y realizando un análisis de datos.

\section{Participantes}

Para esta investigación se aplicó un muestreo no probabilístico intencional o por conveniencia, de esta forma, los sujetos de estudio fueron alumnos del primer año de la carrera profesional de ingeniería civil de dos universidades privadas de la ciudad de Arequipa en el Perú, la Universidad Católica de Santa María (UCSM) y la Universidad Católica San Pablo (UCSP). En ambos casos los alumnos estudiaban por primera vez (no repitentes) los cursos de Geometría Descriptiva (UCSM) y Dibujo Técnico y CAD (UCSP), asignaturas que tenían en general los mismos contenidos: sistemas de representación de objetos, geometría descriptiva del punto, la recta y el plano, intersecciones, poliedros y superficies de revolución y secciones planas; el mismo número de créditos (tres) y seis horas semanales. La diferencia más importante entre ambos cursos fue que en el primero las prácticas eran realizadas exclusivamente a lápiz, mientras que en el segundo, las prácticas eran combinadas con ejercicios a lápiz y ejercicios usando Autocad bidimensional.

Del curso de Geometría Descriptiva (UCSM) se tomó en el semestre 2017-1 a todos los alumnos de dos de las cuatro secciones a las que se tenía acceso por ser el profesor principal de dicho curso y del curso Dibujo Técnico y CAD (UCSP) se tomó en el semestre 2016-2 a todos los alumnos de las cuatro secciones.

Tabla 1: Participantes por sexo y universidad de procedencia.

\begin{tabular}{lccccc}
\hline \multirow{2}{*}{ Universidad } & \multicolumn{2}{c}{ Hombres } & Mujeres & Total \\
\cline { 2 - 6 } & $f$ & $\%$ & $f$ & $\%$ & $f$ \\
\hline Universidad Católica de Santa María & 102 & $75.00 \%$ & 34 & $25.00 \%$ & 136 \\
\hline Universidad Católica San Pablo & 51 & $63.75 \%$ & 29 & $36.25 \%$ & 80 \\
\hline
\end{tabular}

\section{Instrumentos}

Para evaluar la Capacidad Espacial de los estudiantes, se utilizó el Test de Visualización Espacial Purdue: Rotaciones (PSVT: R) (Guay, 1976) que mide específicamente el subcomponente de la Rotación Espacial que es el más representativo y usado (Sorby, 1999). El test consta de 30 ejercicios en los que se muestra un objeto y a continuación el mismo objeto rotado en el espacio, luego se muestra otro objeto diferente y se 
solicita que determine dentro de cinco posibilidades cómo terminaría el segundo objeto si se le aplicara la misma rotación que al primero. Se muestra en la Figura 1 el ejemplo entregado a los estudiantes.

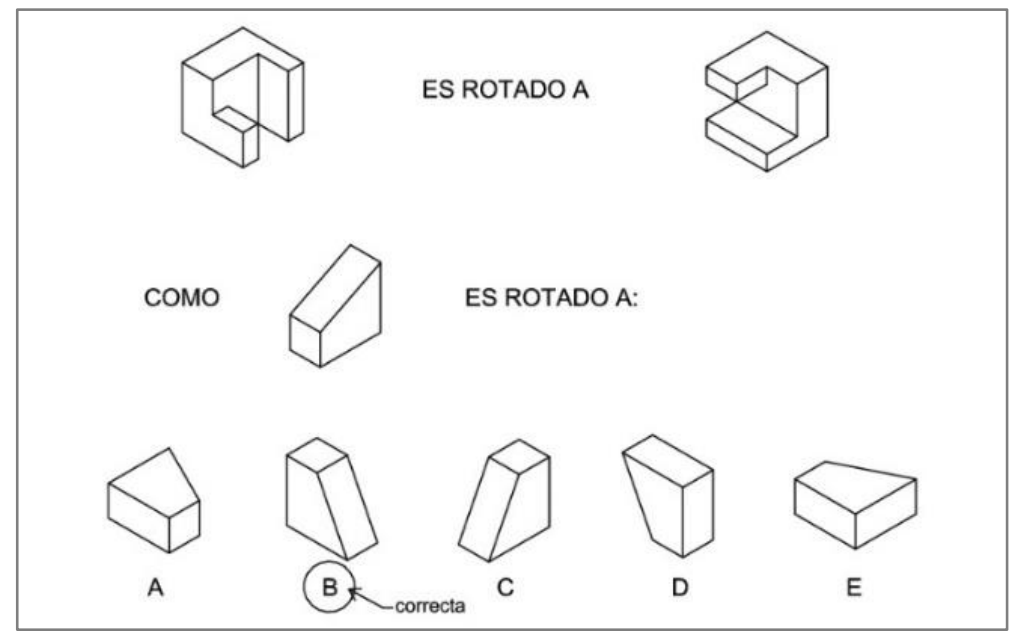

Fig. 1: Ejemplo del Test de Visualización Espacial Purdue: Rotaciones

Para evaluar el rendimiento académico en los cursos semestrales (Geometría Descriptiva o Dibujo Técnico y CAD) se usó la escala vigesimal siendo la nota aprobatoria 12 de 20 puntos en ambas universidades. Los instrumentos utilizados para esas asignaturas fueron exámenes escritos, resolución de ejercicios, trabajos prácticos y controles.

\section{Procedimiento}

Se explicó y solicitó a los estudiantes su consentimiento para formar parte de la investigación, el cual fue declarado firmando en la hoja de identificación, luego se procedió a evaluar la capacidad espacial de los estudiantes con la prueba PSVT: R en la primera semana del semestre para lo cual se les entregó una hoja de identificación con un ejemplo para después otorgarles veinte minutos para solucionar los 30 ejercicios. Luego desde la segunda hasta la decimoquinta semana se impartió el curso (Geometría Descriptiva en UCSM y Dibujo Técnico y CAD en UCSP). Para esta etapa se creó modelos tridimensionales manipulables en tiempo real (archivos PDF en 3D) para cada sesión de aprendizaje, es decir para sistemas de representación de objetos, para la geometría descriptiva del punto (proyección y visibilidades, posiciones relativas, cambio de planos y planos auxiliares), de la recta (proyección, rectas que se cortan y se cruzan, posiciones particulares, verdadera magnitud, orientación, pendiente, paralelismo y perpendicularidad), del plano (proyección y definición, posiciones particulares, verdadera magnitud, orientación y pendiente) y relaciones métricas (ángulos y distancias). De tal forma que para cada explicación teórica se tuvo el apoyo de esta tecnología con la ventaja que permite al docente mover y girar los objetos en tiempo real, y a los estudiantes, relacionar el objeto y sus proyecciones para el mejor entendimiento de la tridimensionalidad de los gráficos bidimensionales como se muestra ejemplos en las figuras 2, 3 y 4. Finalmente para medir el cambio de la capacidad espacial, se volvió a aplicar en la decimosexta semana otra prueba PSVT: R con las mismas condiciones que el primero.


Fig. 2: Ejemplo de un modelo tridimensional (3D-PDF) para explicar los puntos contenidos en una recta. A la izquierda una vista tridimensional, al centro la vista Vertical $(V)$ o frontal y a la derecha la vista Horizontal $(H)$ o superior. 

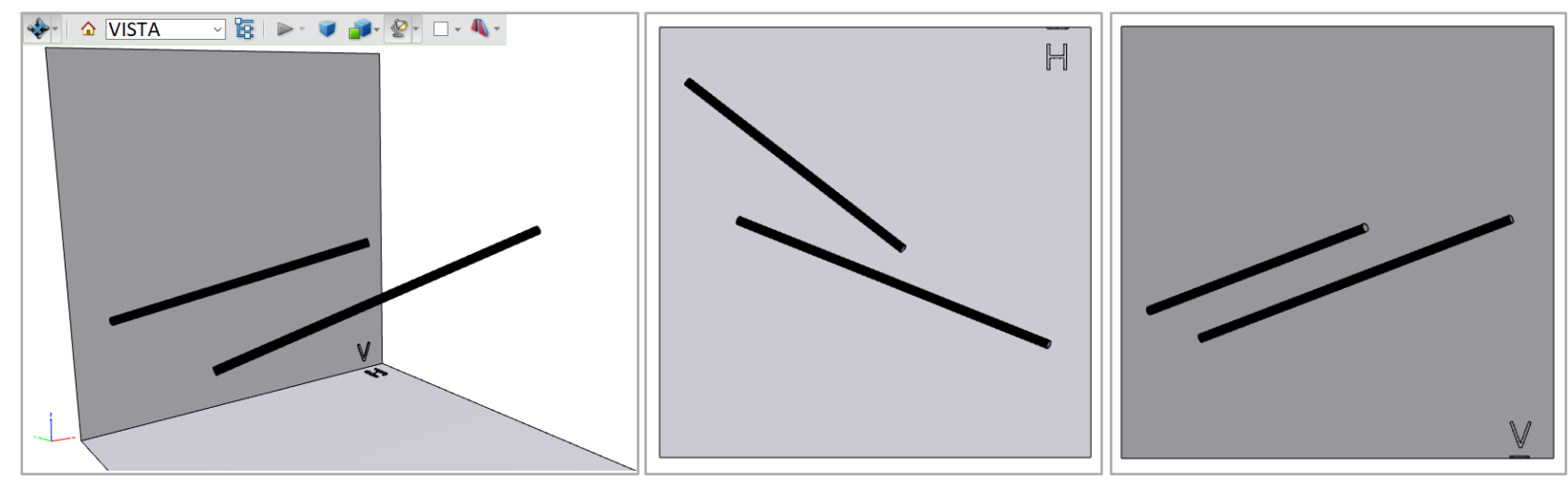

Fig. 3: Ejemplo de un modelo tridimensional (3D-PDF) para determinar el paralelismo entre dos rectas. A la izquierda una vista tridimensional, al centro la vista Vertical $(V)$ o frontal y a la derecha la vista Horizontal $(H)$ o superior.
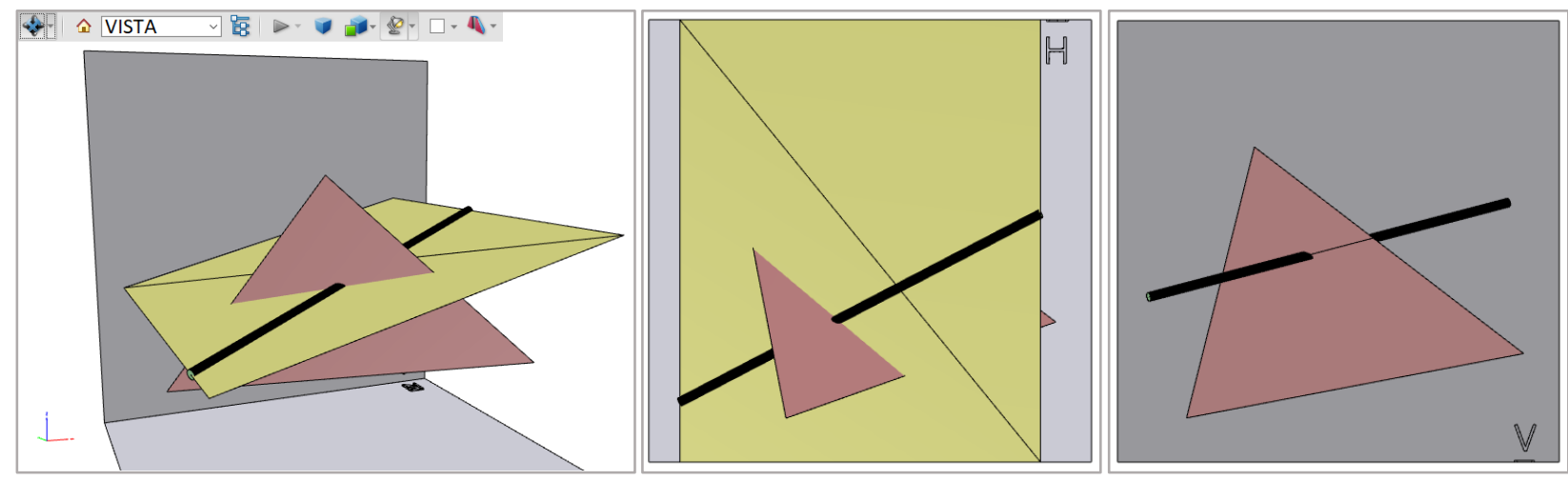

Fig. 4: Ejemplo de un modelo tridimensional (3D-PDF) para explicar el método del Plano Cortante para encontrar la intersección de una recta con un plano. A la izquierda una vista tridimensional, al centro la vista Vertical (V) o frontal y a la derecha la vista Horizontal $(\mathrm{H})$ o superior.

\section{Análisis de datos}

Luego de recolectar la información, se creó una base de datos en el programa especializado para análisis estadístico denominado IBM SPSS en donde se ordenaron los datos con las siguientes condiciones:

Para la prueba PSVT: R, de los treinta ejercicios se considera que una capacidad espacial muy alta corresponde al acierto del $91 \%$ a $100 \%$ (28 a 30 preguntas), alta de $61 \%$ a $90 \%$ (19 a 27 preguntas), baja de $41 \%$ a $60 \%$ ( 13 a 18 preguntas) y muy baja de $0 \%$ a $40 \%$ ( 0 a 12 preguntas). Para el rendimiento académico en los cursos que fue evaluado en la escala vigesimal se considera como nota aprobatoria 12 de 20 puntos en ambas universidades.

Antes de proceder con el análisis inferencial de las variables, se analizó si estas tenían una distribución normal. La prueba de Kolmogorov-Smirnov indicó que la capacidad espacial antes $(\mathrm{K}-\mathrm{S}=.082 ; p=.001)$ y después del entrenamiento $(\mathrm{K}-\mathrm{S}=.112 ; p<.001)$ así como el rendimiento académico en los cursos semestrales $(\mathrm{K}-\mathrm{S}=.178 ; p<.001)$ no siguen una distribución normal. Sin embargo, teniendo en cuenta que este tipo de pruebas tiende a rechazar la hipótesis de normalidad en la mayoría de variables usadas en inteligencia y psicología, se procedió a analizar los datos usando los estadísticos de $Z$ asimetría y $Z$ curtosis, ya que una variable se acercaría a una distribución normal si los valores de ambos estadísticos se encuentran en el rango de [-3.29; 3.29] (Kim, 2013). En nuestro caso se halló que la capacidad espacial antes [Zasimetría= -1.40 ; $\left.Z_{\text {curtosis }}=-1.39\right]$ y después del programa $\left[Z_{\text {asimetría }}=-2.69 ; Z_{\text {curtosis }}=-1.25\right]$ se acercan a una distribución normal por lo cual pueden ser procesadas con estadística paramétrica ( $T$ de Student para muestras independientes). Por otro lado el rendimiento académico debe ser procesado con estadística no paramétrica $\left[Z_{\text {asimetria }}=-6.05 ; Z_{\text {curtosis }}=2.99\right.$ ] ( $U$ de Mann-Whitney).

\section{RESULTADOS}

En la Tabla 2 se muestra la capacidad espacial de los estudiantes antes y después del curso en donde se muestra que la mayoría de estudiantes luego del curso alcanzan un nivel alto (casi la mitad de los que tuvieron un nivel bajo pasaron a este estrato y solo representan ahora un 31.5\%). Al analizar los datos teniendo en 
cuenta el sexo de los evaluados, se encuentra una diferencia importante ya que el $24.9 \%$ de varones mantiene una capacidad espacial post curso baja (comparada con el $49.0 \%$ previo), mientras que el $47.6 \%$ de las mujeres mantienen un nivel bajo (comparada con el $77.8 \%$ previo), es decir se muestra un mayor progreso de estas últimas.

Tabla 2: Capacidad Espacial pre y poscurso

\begin{tabular}{ccccc}
\hline \multirow{2}{*}{$\begin{array}{c}\text { Capacidad } \\
\text { Espacial }\end{array}$} & \multicolumn{2}{c}{ Precurso } & \multicolumn{2}{c}{ Poscurso } \\
\cline { 2 - 5 } & Frecuencia & Porcentaje & Frecuencia & Porcentaje \\
\hline Baja & 124 & 57.40 & 68 & 31.50 \\
Alta & 92 & 42.60 & 148 & 68.50 \\
\hline Total & 216 & 100.00 & 216 & 100.00 \\
\hline
\end{tabular}

En la Tabla 3 se puede observar que existen diferencias estadísticamente significativas entre los varones y las mujeres evaluados en su capacidad espacial tanto antes de la aplicación del curso con gran tamaño del efecto $\left(\mathrm{t}_{(214)}=-5.383 ; p<.001 ; d=0.81\right)$ como después de la aplicación del mismo con un tamaño del efecto moderado $\left(\mathrm{t}_{(214)}=-4.136 ; p<.001 ; d=0.62\right)$, en ambos casos son los varones quienes presentan una mayor capacidad espacial que sus pares mujeres. Por otro lado, no se encontró diferencias estadísticamente significativas, entre los alumnos de una universidad y la otra, en su capacidad espacial antes del curso ( $\mathrm{t}(214)=$ -0.048; $p=.962)$, ni después de terminado el mismo $\left(\mathrm{t}_{(214)}=1.026 ; p=.306\right)$.

Tabla 3: Diferencias de la Capacidad Espacial según sexo y universidad de procedencia.

\begin{tabular}{|c|c|c|c|c|c|c|c|c|c|c|}
\hline & & \multirow{2}{*}{$N$} & \multirow{2}{*}{ Media } & \multirow{2}{*}{$\begin{array}{l}\text { Desviación } \\
\text { estándar }\end{array}$} & \multicolumn{2}{|c|}{ Prueba de Levene } & \multirow{2}{*}{$t$} & \multirow{2}{*}{$g l$} & \multirow{2}{*}{$\begin{array}{c}\text { Sig. } \\
\text { (bilateral) }\end{array}$} & \multirow{2}{*}{$\begin{array}{l}d d e \\
\text { Cohen }\end{array}$} \\
\hline & & & & & $F$ & Sig. & & & & \\
\hline \multirow[t]{2}{*}{ CE precurso } & Mujer & 63 & 14.38 & 5.122 & 1.615 & 0.205 & -5.383 & 214 & $<0.001$ & 0.81 \\
\hline & Varón & 153 & 18.19 & 4.555 & & & & & & \\
\hline \multirow[t]{2}{*}{ CE poscurso } & Mujer & 63 & 18.86 & 5.121 & 9.011 & 0.003 & -3.726 & 214 & $<0.001$ & 0.62 \\
\hline & Varón & 153 & 21.54 & 3.974 & & & & & & \\
\hline \multirow[t]{2}{*}{ CE precurso } & UCSM & 136 & 17.07 & 5.066 & 0.070 & 0.791 & -0.048 & 214 & 0.962 & - \\
\hline & UCSP & 80 & 17.10 & 4.985 & & & & & & \\
\hline \multirow[t]{2}{*}{ CE poscurso } & UCSM & 136 & 21.00 & 4.234 & 3.557 & 0.061 & 1.026 & 214 & 0.306 & - \\
\hline & UCSP & 80 & 20.35 & 4.912 & & & & & & \\
\hline
\end{tabular}

En la Tabla 4 se aprecia que existen diferencias significativas en la capacidad espacial luego de aplicar el curso $\left(\mathrm{t}_{(215)}=-13.490 ; p<.001 ; d=0.77\right)$, dicho incremento tiene un tamaño del efecto moderado, que se corresponde con un $22 \%$ de incremento en la capacidad espacial de los alumnos. Haciendo la comparación por sexos, se halló que estas diferencias significativas en las alumnas luego de aplicar el curso $\left(\mathrm{t}_{(62)}=-8.070\right.$; $p<.001 ; d=0.88)$, muestran un tamaño del efecto grande en la mejoría, que se corresponde con un $31 \%$ de incremento en la capacidad espacial. Mientras que en los hombres $\left(t_{(152)}=-10.904 ; p<.001 ; d=0.79\right)$ dicha mejoría tiene un tamaño del efecto moderado, que se corresponde con un $18 \%$ de incremento en la capacidad espacial.

En cuanto a la universidad de procedencia, se encontró que existen diferencias significativas en la capacidad espacial de los alumnos evaluados de la UCSM luego del curso $\left(\mathrm{t}_{(135)}=-10.497 ; p<.001 ; d=0.84\right)$, mostrando un incremento que representa un tamaño del efecto grande $(23 \%)$ mientras que en los alumnos de la UCSP

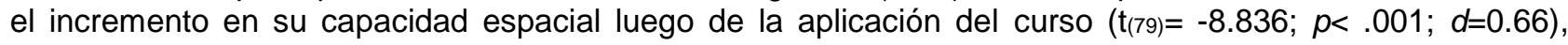
muestra una mejoría que tiene un tamaño del efecto moderado (19\%).

Tabla 4: Diferencias de la Capacidad Espacial antes y después del curso.

\begin{tabular}{ccccccc}
\hline \multirow{N}{*}{ =216 } & Media & $\begin{array}{c}\text { Desviación } \\
\text { estándar }\end{array}$ & $t$ & $g l$ & $\begin{array}{c}\text { Sig. } \\
\text { (bilateral) }\end{array}$ & $\begin{array}{c}\text { dde } \\
\text { Cohen }\end{array}$ \\
\hline Capacidad espacial precurso & 17.08 & 5.024 & -13.490 & 215 & $<0.001$ & 0.77 \\
Capacidad espacial poscurso & 20.76 & 4.497 & & & & \\
\hline
\end{tabular}


En la tabla 5 se analiza si el curso tuvo mayor impacto en los que inicialmente tenían capacidad baja o alta y se encontró que en los alumnos que tuvieron una capacidad espacial baja $\left(\mathrm{t}_{(123)}=-14.623 ; p<.001 ; d=1.32\right)$, su incremento luego de pasar por el curso, tuvo un tamaño del efecto muy grande, que se corresponde con un 37\%; mientras que al analizar solo los casos de aquellos alumnos que tenían previamente una capacidad espacial alta $\left(\mathrm{t}_{(91)}=-5.075 ; p<.001 ; d=0.61\right)$, su incremento tuvo un tamaño del efecto pequeño que se corresponde con un $8 \%$. Esto nos indica que el curso fue más efectivo para los alumnos de capacidad espacial baja.

Tabla 5: Impacto del curso en alumnos con bajo y alto nivel de Capacidad Espacial.

\begin{tabular}{|c|c|c|c|c|c|c|c|}
\hline & & Media & $\begin{array}{l}\text { Desviación } \\
\text { estándar }\end{array}$ & $t$ & $g l$ & $\begin{array}{c}\text { Sig. } \\
\text { (bilateral) }\end{array}$ & $\begin{array}{l}\text { dde } \\
\text { Cohen }\end{array}$ \\
\hline \multirow{2}{*}{$\begin{array}{l}\text { Capacidad baja } \\
(\mathrm{N}=124)\end{array}$} & Capacidad espacial precurso & 13.67 & 3.489 & -14.623 & 123 & $<0.001$ & 1.32 \\
\hline & Capacidad espacial poscurso & 18.77 & 4.208 & & & & \\
\hline \multirow{2}{*}{$\begin{array}{l}\text { Capacidad alta } \\
-(\mathrm{N}=92)\end{array}$} & Capacidad espacial precurso & 21.67 & 2.450 & -5.075 & 91 & $<0.001$ & 0.61 \\
\hline & Capacidad espacial poscurso & 23.45 & 3.336 & & & & \\
\hline
\end{tabular}

Por último, se encontró que la capacidad espacial tiene una relación estadísticamente significativa con el rendimiento académico, es decir con el promedio final que obtuvieron los alumnos en el curso semestral de la carrera (Rho de Spearman, $r=.381 ; p<.001$ ), dicha relación es directamente proporcional, lo que nos indica que una mayor capacidad espacial se asocia con un mayor rendimiento en estos cursos de graficación en ingeniería.

\section{DISCUSIÓN}

El impacto de incorporar una herramienta tecnológica, como los modelos tridimensionales manipulables en tiempo real (archivos 3D-PDF), para la mejor explicación de los contenidos teóricos de la Geometría Descriptiva en los alumnos del primer año de Ingeniería Civil y la posibilidad de constituirse en un entrenamiento indirecto de larga duración de la capacidad espacial, ha sido moderado. Estos hallazgos contribuyen a fortalecer la tendencia que considera que los entrenamientos indirectos que combinan la ingeniería gráfica tradicional con las tecnologías informáticas (el modelado y visualización tridimensional) favorecen en mayor medida el desarrollo de la capacidad espacial que otros (Marunić y Glažar, 2014) y que el uso que los modelos 3D-PDF, a diferencia de la realidad virtual y aumentada, facilitan las tareas de entrenamiento en grupos numerosos, además de ser de bajo costo, fáciles de crear y manejar y de su disponibilidad permanente.(Domínguez et al., 2012).

Se esperaba un impacto mayor, sin embargo no sucedió probablemente porque el apoyo de los modelos tridimensionales se centraron en lograr el mejor entendimiento de los temas propios de la Geometría Descriptiva, siendo muchos de ellos de naturaleza abstracta y difícil entendimiento, más que un entrenamiento específico de la capacidad espacial. Para lograr un mayor impacto se propone investigar el efecto de modelos 3D-PDF combinando los modelos propuestos con modelos de objetos cotidianos como lo propone la ENGAGE Engineering (Metz, 2015).Y también se propone estudiar el efecto de modelos de realidad aumentada (RA), pero que al igual que lo propuesto en esta investigación, estén orientados a cada uno de los temas propios de la Geometría Descriptiva y que están demostrando una efectividad casi tan alta como los 3D-PDF en el desarrollo de la capacidad espacial (Martin-Gutiérrez et al).

Los resultados muestran que el entrenamiento de esta investigación tiene el mismo nivel de impacto que otros que también usaron en su metodología los archivos 3D-PDF (Martin-Gutiérrez et al., 2013 y Leopold et al., 2001), sin embargo, la importancia y ventaja de este radica en que es un entrenamiento indirecto y no específico, es decir, que ha usado el espacio y tiempo académicos de una asignatura obligatoria del primer año (no ha demandado laboratorios, créditos o costos extracurriculares) y que se ha concentrado en cada tema propio de la asignatura (no solo en la visualización de piezas tridimensionales). En ese sentido, la metodología planteada es un aporte a la enseñanza superior puesto que es menester adaptarse y utilizar las nuevas tecnologías dejando atrás aquellas obsoletas para el beneficio de los estudiantes y el aprendizaje universitario (Martin-Gutiérrez et al., 2013).

De otro lado, dado el porcentaje alto de estudiantes con capacidad espacial muy baja (19\%), condición que se repite en la mayoría de carreras de ingeniería en el Perú, se recomienda investigar entrenamientos directos, específico y de corta duración que según Veurink y Sorby (2017) tienen un efecto mucho mayor en el desarrollo de la capacidad espacial; diseños experimentales que tendrán que considerar grupos de control (limitación importante del estudio) para proporcionar argumentos más contundentes que expresen la relación 
de causa y efecto. En cuanto a género, y coincidiendo con las investigaciones que muestran un predominio de la capacidad espacial de los hombres sobre las mujeres (Nagy-Kondor, 2016, Maeda y Yoon, 2013), los hallazgos han contribuido en el mismo sentido, sin embargo se propone indagar más al respecto ya que según Veurink y Sorby (2017) no es muy claro el tipo de entrenamiento deben tener las mujeres jóvenes y si es mejor en entrenamiento a edad temprana.

La investigación plantea la importancia de conocer, estudiar, evaluar, desarrollar y entrenar en el Perú la Capacidad Espacial de todos aquellos estudiantes que quieran contribuir con la ciencia, la tecnología, la ingeniería y las matemáticas en el Perú, de tal forma que esta capacidad se convierta en un predictor de éxito para los futuros ingenieros, fortaleciendo así el capital humano para el desarrollo tecnológico de la región.

\section{CONCLUSIONES}

De acuerdo a los resultados y discusión de esta investigación se tienen cinco conclusiones: 1) la característica principal de la metodología se concentra en las explicaciones tridimensionales de los temas propios de la geometría descriptiva buscando la mejor visualización y entendimiento de los elementos en el espacio por parte de los estudiantes; 2) el nivel de la Capacidad Espacial con que la mayoría de estudiantes (57.4\%) ingresan a estudiar la carrera de Ingeniería Civil en Arequipa es bajo, pero además, un quinto (19.0\%) ingresa con una capacidad muy baja; 3) la utilización de los archivos 3D-PDF en ambos cursos, los convierte en un entrenamiento indirecto de largo plazo para la Capacidad Espacial de impacto moderado (22\% de incremento); 4) el efecto del entrenamiento en la Capacidad Espacial de los estudiantes es más satisfactorio en las mujeres (incremento del $31 \%$ comparado con $18 \%$ de los hombres) y en los que presentaron inicialmente una capacidad baja (incremento del $38 \%$ comparado con $8 \%$ de los de alta capacidad) y 5 ) en Arequipa un entrenamiento indirecto no es suficiente para lograr un desarrollo óptimo y mayoritario de esa capacidad tan importante, haciéndose necesario un entrenamiento directo y específico.

\section{AGRADECIMIENTOS}

Se agradece a la Universidad Católica de Santa María y a la Universidad Católica San Pablo por permitir el desarrollo de esta investigación y a los alumnos del primer año de Ingeniería Civil de ambas universidades por su colaboración.

\section{REFERENCIAS}

Acevedo, D., J. D. Torres y M. J. Jiménez, Factores Asociados a la Repetición de Cursos y Retraso en la Graduación en Programas de Ingeniería de la Universidad de Cartagena, en Colombia, doi: 10.4067/S0718-50062015000200006, Formación Universitaria, 8(2), 35-42 (2015)

Alqahtani, A.S., L F. Daghestani y L. F. Ibrahim, Techniques used to Improve Spatial Visualization Skills of Students in Engineering Graphics Course: A Survey, doi: 10.14569/IJACSA.2017.080315, International Journal of Advanced Computer Science and Applications (IJACSA), 8(3), 91-100 (2017)

Arrieta, I. y M. C. Medrano, Un Análisis de la Capacidad Espacial en Estudios de Ingeniería Técnica, PNA, ISSN: 18873987, Revista de Investigación en Didáctica de La Matemática, 9(2), 85-106 (2015)

Arrieta, M., La Capacidad Espacial en la Educación Matemática: Estructura y Medida, Educación Matemática 18(1), 99$132(2006)$

Baenninger, M. y N. Newcombe, The Role of Experience in Spatial Test Performance: A Meta-Analysis, doi: 10.1007/BF00287729, Sex Roles, 20(5-6), 327-344 (1989)

$\mathrm{CEH}$, Committee on Education and Human Resources. Preparing the Next Generation of STEM Innovators: Identifying and Developing our Nation's Human Capital, 15-18, N.S. Board, Virginia, USA (2010)

Contreras, L.E., J.A. Trisancho y L. F. Vargas, Evaluación de Factores de Entorno que afectan el Desarrollo de Habilidades Espaciales en Estudiantes de Primer Semestre en Ingeniería Industrial, doi:10.18359/ravi.1921, Revista Academia y Virtualidad 6(1), 17 (2013)

Domínguez, M. G., J. Martin-Gutierrez, C. R. Gonzalez y C. M. M. Corredeaguas, Methodologies and Tools to Improve Spatial Ability, doi: 10.1016/j.sbspro.2012.08.233, Procedia-Social and Behavioral Sciences, 51, 736-744 (2012)

Guay, R. y Purdue Research Foundation, Purdue Spatial Visualization Test, 1ra edición, 53-71, West Layfette, Ind., Purdue University, Indiana, USA (1976)

Kim, H.-Y., Statistical Notes for Clinical Researchers: Assessing Normal Distribution (2) using Skewness and Kurtosis, doi: 10.5395/rde.2013.38.1.52, Restorative Dentistry \& Endodontics, 38(1), 52-54 (2013)

Kösa, T. y F. Karakuş, The Effects of Computer-Aided Design Software on Engineering Students' Spatial Visualisation Skills, doi: 10.1080/03043797.2017.1370578, European Journal of Engineering Education, 43(2), $296-308$ (2018) 
Leopold, C., R. A. Gorska y S.A. Sorby, International Experiences in Developing the Spatial Visualization Abilities of Engineering Students, ISSN:1433-8157, Journal for Geometry and Graphics, 5(1), 81-91 (2001)

Maeda, Y. y S. Y. Yoon, A Meta-Analysis on Gender Differences in Mental Rotation Ability Measured by the Purdue Spatial Visualization Tests: Visualization of Rotations (PSVT: R), doi: 10.1007/s10648-012-9215-x, Educational Psychology Review, 25(1), 69-94 (2013)

Martin-Gutierrez, J., M., Garcia-Dominguez, C. R. Gonzalez y M. M. Corredeguas, Using Different Methodologies and Technologies to Training Spatial Skill in Engineering Graphic Subjects. Frontiers in Education Conference, IEEE, 362-368, Oklahoma, USA 23-26 Octubre (2013)

Marunić, G. y V. Glažar, Improvement and Assessment of Spatial Ability in Engineering Education, ISSN: 1849-0433, Engineering Review, 34(2) 139-150 (2014).

Metz, S., International Innovation: Disseminating Science, Research and Technology, US National Science Foundation (2015)

Nagy-Kondor, R., Gender Differences in Spatial Visualization Skills of Engineering Etudents, Annales Mathematicae et Informaticae, 46, 265-276 (2016)

Pieterse, F. y A. Nel, Teaching Graphical Communication to first year Engineering Students. Global Engineering Education Conference (EDUCON), IEEE. 405-409, Berlin, Germany 13-15 Marzo (2013)

Saorín-Pérez, J. L., R. E. Navarro-Trujillo, N.Martín-Dorta, J. Martín-Gutiérrez y M. Contero, La Capacidad Espacial y su Relacion con la Ingeniería, doi: http://dx.doi.org/10.6036/2870, Dyna 84(9), 721-732 (2009)

Segil, J. L., J. F. Sullivan, J. Y.Tsai, D.T. Reamon y M. H. Forbes, Investigation of Spatial Visualization Skills Across World Regions. 2017 IEEE Frontiers in Education Conference (FIE), 1-5, Indianapolis, USA 18-21 Oct. (2017)

Sorby, S., E. Nevin, A. Behan, E. Mageean y S. Sheridan, Spatial Skills as Predictors of Success in First-Year Engineering. Frontiers in Education Conference (FIE), 1-7, Madrid, Spain 22-25 Oct. (2014)

Sorby, S. A., Developing 3-D Spatial Visualization Skills, The Engineering Design Graphics Journal, 63(2) (1999)

Sorby, S. A., Developing 3D Spatial Skills for Engineering Students, doi: 10.1080/22054952.2007.11463998, Australasian Journal of Engineering Education, 13(1), 1-11 (2007)

Sorby, S.A., Educational Research in Developing 3-D Spatial Skills for Engineering Students, doi: 10.1080/09500690802595839, International Journal of Science Education, 31(3), 459-480 (2009)

Sorby, S. A. y B. J. Baartmans, The Development and Assessment of a Course for Enhancing the 3-D Spatial Visualization Skills of First Year Engineering Students, doi: 10.1002/j.2168-9830.2000.tb00529.x, Journal of Engineering Education 89(3), 301-307 (2000)

Tartre, L.A., Spatial Orientation Skill and Mathematical Problem Solving, doi: 10.2307/749375, Journal for Research in Mathematics Education, 21(3), 216-229 (1990)

Tristancho, J.A., L.E. Contreras y L.F. Vargas, Evaluación de Técnicas Tradicionales y TIC para el Desarrollo de Habilidades Espaciales en Estudiantes de primer semestre de Ingeniería Industrial, ISSN: 0124-5821, Revista Virtual Universidad Católica del Norte, 4(43), 34-50 (2014)

Veurink, N. L. y S. A. Sorby, Longitudinal Study of the Impact of Requiring Training for Students with Initially Weak Spatial Skills, doi: 10.1080/03043797.2017.1390547, European Journal of Engineering Education, 1-11 (2017)

Wai, J., D. Lubinski y C. P. Benbow, Spatial Ability for STEM Domains: Aligning over 50 years of Cumulative Psychological Knowledge Solidifies its Importance, doi: 10.1037/a0016127, Journal of Educational Psychology 101(4), 817 (2009) 
